\title{
INTERVENCIÓN PSICOLÓGICA COMO PARTE DEL TRATAMIENTO INTEGRAL DE PACIENTES CON INSUFICIENCIA CARDÍACA: EFECTO SOBRE LA CAPACIDAD FUNCIONAL
}

\author{
VIRIDIANA PELÁEZ HERNÁNDEZ*, ARTURO OREA-TEJEDA**, BENJAMÍN DOMÍNGUEZ TREJO***, \\ CARLOS GONZALO FIGUEROA LÓPEZ ${ }^{* * * *, ~ L U I S ~ A L C O C E R ~ D I ́ A Z ~ B A R R E I R O * * * *, ~}$ \\ RUTH PABLO SANTIAGO ${ }^{\star * * * * *}$, CLAUDIA IVONN MARTÍNEZ RUBIO******* \\ INSTITUTO NACIONAL DE ENFERMEDADES RESPIRATORIAS “ISMAEL COSÍO VILLEGAS" - \\ UNIVERSIDAD NACIONAL AUTÓNOMA DE MÉXICO. CIUDAD DE MÉXICO, MÉXICO.
}

Recibido: 14 de Septiembre de 2015

Aprobado: 16 de Marzo de 2016

Para referenciar este artículo:

Peláez Hernández V., Orea Tejeda, A., Domínguez-Trejo, B., Figueroa López, C., Díaz Barreiro, L., Santiago, R. \& Martínez Rubio, C. (2016). Intervención psicológica como parte del tratamiento integral de pacientes con insuficiencia cardíaca: efecto sobre la capacidad funcional Revista Iberoamericana de Psicología: Ciencia y Tecnología, 9 (1), 53-61

\begin{abstract}
Resumen
El objetivo del presente trabajo fue investigar el efecto de una intervención psicológica cognitivo-conductual en la capacidad funcional de los pacientes con insuficiencia cardiaca, medida a través de la prueba de caminata de seis minutos. El estudio contó con la participación de 53 pacientes con insuficiencia cardiaca de clases funcionales I y II, los cuales fueron asignados aleatoriamente a: 1) grupo control (tratamiento médico usual, n=27, edad media 61,07 $\pm 15,96$; $55,6 \%$ hombres); o 2) grupo experimental (tratamiento médico usual e intervención psicológica, n=26, edad media $58,42 \pm 16,42 ; 65,4 \%$ mujeres). Mediante la prueba de caminata de seis minutos se evaluó la distancia recorrida en metros, el nivel de cansancio físico y el nivel de esfuerzo reportado en la escala de Borg. Las evaluaciones se realizaron antes y al final del programa de intervención, y se realizó un seguimiento que se extendió durante los siguientes tres meses. El programa de intervención psicológica incluyó psico-educación, respiración diafragmática, relajación muscular progresiva y entrenamiento en solución de problemas en cuatro sesiones de 90 minutos, diferidas semanalmente. Los resultados muestran que mientras el grupo experimental disminuyó el nivel de cansancio físico $(p=0,02)$ y el nivel de esfuerzo reportados (ns) cuando incrementó la distancia recorrida $(p=0,00)$, el grupo control no mostró cambios significativos. Se concluye que la intervención propuesta tiene un efecto positivo sobre la condición de salud de los pacientes, en tanto se adapta a las necesidades específicas de la población; por ello se sugiere la integración de equipos interdisciplinarios que incluyan programas de intervención psicológica para mejorar la salud cardiovascular y la calidad de vida de los pacientes.

Palabras clave: Cardiología conductual, tratamiento no-farmacológico, estrés físico, prueba de esfuerzo.
\end{abstract}

* Investigadora principal. Doctora en Psicología. Coordinadora de Proyectos de Investigación en Psicología del Servicio de Cardiología del Instituto Nacional de Enfermedades Respiratorias "Ismael Cosío Villegas". E- mail: psic.viridiana.pelaez@gmail.com

** Especialista en Cardiología. Coordinador del Servicio de Cardiología del Instituto Nacional de Enfermedades Respiratorias "Ismael Cosío Villegas". E- mail: oreatart@gmail.com

*** Doctor en Psicología. Docente investigador de la División de Investigación y Posgrado de la Facultad de Psicología, Universidad Nacional Autónoma de México.

**** Doctor en Psicología. Facultad de Estudios Superiores Zaragoza, Universidad Nacional Autónoma de México.

$* * * * * *$ Licenciada en Psicología. Miembro de la Clínica de Insuficiencia Cardiaca y Respiratoria del Instituto Nacional de Enfermedades Respiratorias "Ismael Cosío Villegas".

$* * * * * *$ Psicóloga. Pasante en la Clínica de Insuficiencia Cardiaca y Respiratoria del Instituto Nacional de Enfermedades Respiratorias "Ismael Cosío Villegas". 


\title{
PSYCHOLOGICAL INTERVENTION AS PART OF COMPREHENSIVE TREATMENT OF HEART FAILURE PATIENTS: EFFECT ON FUNCTIONAL CAPACITY
}

\begin{abstract}
The aim of this study was to investigate the effect of a cognitive-behavioral psychological intervention on the functional capacity of heart failure patients, effect measured by the six minute walk test. Fifty three patients with heart failure -functional class I and II- participated, who were randomly assigned to: 1 ) the control group (usual medical treatment, $\mathrm{n}$ $=27$, mean age $61.07 \pm 15.96,55.6 \%$ male), or 2 ) the experimental group (usual medical treatment and psychological intervention, $\mathrm{n}=26$, mean age $58.42 \pm 16.42,65.4 \%$ women). By means of the six-minute walk test, the distance traveled in meters, the physical fatigue level and effort level reported in the Borg scale, were all assessed. The evaluations were performed both before and at the end of the intervention program, plus a three-month follow-up was carried out. The psychological intervention program included psycho-education, diaphragmatic breathing, progressive muscle relaxation and training in problem solving, in four sessions of 90 minutes each, deferred weekly. The results show that while the experimental group decreased the reported physical fatigue level $(p=0.02)$ and the effort level (ns) when the traveled distance increased $(p=0.00)$, the control group showed no significant changes. We concluded that the proposed intervention has a positive effect on the health condition of patients, as it is tailored to the special needs of this population; thus we suggest the integration of interdisciplinary teams that include psychological intervention programs to improve cardiovascular health and the quality of life of patients.

Keywords: Behavioral Cardiology, non-pharmacological treatment, physical stress, effort test.
\end{abstract}

La Insuficiencia Cardiaca (IC) es una característica común a las etapas finales de gran parte de las enfermedades cardiovasculares. Consiste en un síndrome clínico complejo en el que la capacidad del corazón para mantener la circulación sanguínea adecuada a las necesidades metabólicas del organismo se halla limitada, lo cual conduce a que se produzcan síntomas y signos específicos como disnea, fatiga y edemas, tanto en estado de reposo como al hacer ejercicio (Dornelas, 2008; Hunt et al., 2009; McMurray et al., 2012). A la fecha, la IC muestra características de epidemia que se reflejan en la mortalidad, la pérdida de años productivos, el aislamiento psicosocial y, como consecuencia, el incremento en el uso y los costos para los sistemas de salud (Achury, 2007; Asensio, Orea, Castillo, Dorantes, y Oseguera, 2005; Orea y Jiménez, 2005).

Hoy día sabemos que entre 1 y $2 \%$ de la población adulta de países desarrollados padece IC y que se presentan cerca de 550.000 casos nuevos anuales (Alfaro et al., 2007; McMurray et al., 2012; Roger et al., 2012). En México, aunque hay subregistro de morbi-mortalidad, se estima una prevalencia de 750.000 casos y una incidencia de 75.000 nuevos casos anuales. También sabemos que solo $25 \%$ de los hombres y $38 \%$ de las mujeres sobreviven después de cinco años de padecer el síndrome (Alfaro et al., 2007; Asensio et al., 2005; Orea y Jiménez, 2005).

Con base en la capacidad para realizar actividad física limitada por disnea, la New York Heart Association (NYHA) clasifica la IC en cuatro clases funcionales (McMurray et al., 2012):
I) Sin síntomas con actividades ordinarias y sin limitación para actividad física.

II) Sin síntomas en reposo, pero con limitaciones leves a la actividad física; aparece disnea con esfuerzos intensos.

III) Sin síntomas en reposo, pero con limitación importante para la actividad física; aparece disnea con los esfuerzos medianos.

IV) Síntomas en reposo e incapacidad para realizar cualquier actividad.

Adicionalmente, las limitaciones funcionales que impone la IC afectan diversos aspectos de la vida de los pacientes, razón por la cual la tendencia en intervención ha apuntado a ofrecer tratamientos interdisciplinarios que busquen una mejoría significativa de la calidad de vida, reduzcan las causas de mortalidad, las hospitalizaciones y, si es el caso, aumenten las expectativas de supervivencia (Asensio et al., 2005). La incorporación de intervenciones psicológicas a los procedimientos estándares de rehabilitación cardiaca, por ejemplo, ha demostrado reducir morbilidad, la recurrencia de episodios coronarios, las hospitalizaciones, la mortalidad y el estrés; además ha mostrado efectos positivos en la adherencia al tratamiento y la modificación de factores de riesgo biológico como hipertensión arterial, frecuencia cardiaca y colesterol elevados (Arrivillaga, Varela, Cáceres, Correa y Orlguín, 2007; Cerezo, Hernández y Rodríguez, 2009; Del Pino, Gaos, Dorta y García, 2004; Kulur, Haleagrahara, Adhikary 
\& Jeganathan, 2008; Reyes, 2011). No obstante, aun a pesar del potencial que encierra la incorporación de ese tipo de intervenciones como complemento del tratamiento farmacológico y quirúrgico, la situación real es que hoy día el tratamiento psicológico no está contemplado en las guías de práctica clínica para el tratamiento de estos pacientes (Jiménez, Luna y Huertas, 2009; McMurray et al., 2012).

Los desarrollos interventivos impulsados al interior de la disciplina psicológica han tomado como punto principal punto de referencia al modelo cognitivo-conductual. Pero, aunque ese modelo sea uno de los que cuenta con mayor evidencia científica a su favor, aun no se ha comprobado su eficacia en la modificación de la capacidad funcional de esta población (Alarcón y Ramírez, 2006; Dornelas, 2008). Por tal razón, el objetivo del presente estudio fue evaluar los efectos sobre la capacidad funcional de pacientes con insuficiencia cardiaca a través de una intervención psicológica de corte cognitivo-conductual, basada en la prueba de caminata de seis minutos. Esta prueba consiste en un método simple, fácil de aplicar y de bajo costo, que permite evaluar objetivamente las limitaciones funcionales $y$ el pronóstico de los pacientes con IC, así como el progreso de la terapia y la evolución clínica de los mismos.
En esta prueba se pide al paciente que camine (sin sentir molestias) durante seis minutos para, posterior a este tiempo, medir los metros recorridos (Jefferson, Santos, Paulo \& Brofman, 2008; Rodríguez y Rodríguez, 2005; Rubin, 2012). La distancia caminada es altamente reproducible, tiene valor pronóstico de mortalidad/ hospitalizaciones y está asociada positivamente con la calidad de vida (Arslan et al., 2007; Forman et al., 2012; Guimarães, Carvalho \& Bocchi, 2008; Jefferson et al., 2008; Rodríguez y Rodríguez, 2005). Una revisión previa sobre el uso de la prueba permitió notar que se recomienda su utilización para llevar a cabo evaluación funcional y para estimar el pronóstico de los pacientes con IC pues, según pruebas médicas basadas en evidencia experimental, se estima que cuenta con un nivel de recomendación B para el desarrollo de intervenciones (Arslan et al., 2007).

\section{Método}

Se llevó a cabo un estudio longitudinal, de tipo pre-test/ pos-test con grupo control y seguimiento a tres meses, entre enero de 2013 y febrero de 2014, tal y como se ilustra en la Figura 1. La investigación se llevó a cabo conforme a los lineamientos éticos expresados en la Declaración de Helsinky de 1975.

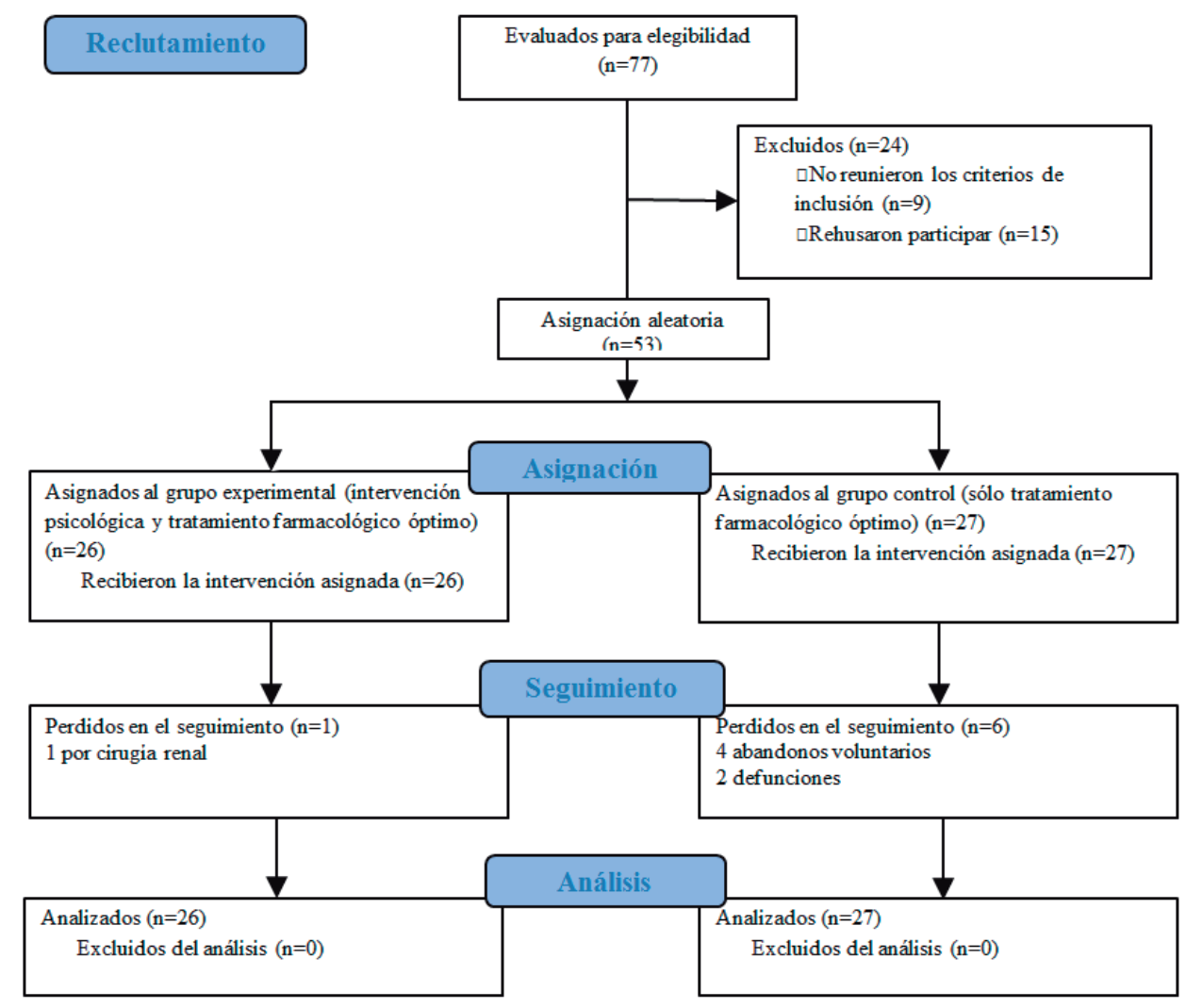

Figura 1. Estructura básica del estudio 


\section{Participantes}

Los pacientes se contactaron en una Clínica de Insuficiencia Cardiaca en la Ciudad de México. La muestra incluyó pacientes con IC, específicamente de clases funcionales I y II de acuerdo con los criterios de la NYHA (McMurray et al., 2012).

Los criterios de inclusión fueron: Pacientes de cualquier género, mayores de 18 años, tiempo de diagnóstico menor a cinco años, en tratamiento farmacológico óptimo y participación voluntaria. Se excluyeron todos aquellos pacientes con implante de dispositivo cardiaco, diabetes mellitus descontrolada o secuelas, hipertensión arterial descontrolada, infección aguda (estomacal, respiratoria, etc.), sordera o diagnóstico psiquiátrico.

\section{Equipo e instrumentos}

Para obtener los datos de los participantes se utilizó una ficha de Identificación que especificaba información acerca de datos sociodemográficos, datos clínicos y los resultados de la prueba de caminata de seis minutos de cada paciente. Durante la realización de la prueba de caminata se emplearon dos escalas:

a) Nivel subjetivo de cansancio físico: Mide el nivel de cansancio que experimenta el paciente al inicio y al término de la prueba. Se trata de una escala tipo likert que va de 1 (sin cansancio o fatiga) a 5 (muy cansado o fatigado).

b) Escala de Borg: Evalúa el nivel de esfuerzo que representa al paciente al hacer la prueba de la caminata de seis minutos. Es una escala tipo likert que va de 0 (nada de esfuerzo) a 10 (esfuerzo extremadamente pesado).

\section{Procedimiento}

Un médico cardiólogo realizó una evaluación previa cuyo objetivo era verificar los datos clínicos de los pacientes, determinar si cumplían con los criterios diagnósticos de IC clase funcional I o II, y cerciorarse sobre la factibilidad de la aplicación de acuerdo con las condiciones particulares de cada paciente. En una sesión individual de aproximadamente 20 minutos se informaba al paciente sobre el objetivo del estudio, se le invitaba a participar y si aceptaba se le pedía firmar la Carta de Consentimiento Informado. En caso contrario, se excluía de la muestra, sin que esto acarreara sanción alguna por parte de la institución.

Cuando el paciente aceptaba participar en la investigación, se procedía a la resolución de la ficha de identificación y la aplicación de la prueba. Al iniciar la prueba, se pedía al paciente que caminara durante seis minutos, hasta donde su condición física lo permitiera, previniéndole de no correr y de permanecer en silencio; no se brindaba indicación adicional alguna. Al terminar, el paciente tomaba asiento e inmediatamente se evaluaba el nivel subjetivo de cansancio físico: mediante la Escala de Borg el paciente indicaba cuánto esfuerzo le representó hacer la prueba de la caminata de seis minutos. Asimismo, se tomó registro de la distancia recorrida por cada paciente.

A continuación, los participantes fueron divididos aleatoriamente en dos grupos: 1) Grupo Control (GC): únicamente recibieron tratamiento farmacológico óptimo; 2) Grupo Experimental (GE): recibieron tratamiento farmacológico óptimo y asistieron a la intervención psicológica.

El tratamiento psicológico se impartió en un aula asignada dentro del Instituto Nacional de Salud en Ciudad de México. Constó de cuatro sesiones grupales de 90 minutos diferidas semanalmente, e incluyó las siguientes técnicas:

- Técnicas de relajación: Respiración diafragmática y relajación muscular progresiva: La primera consiste en entrenar al paciente para respirar profundamente con su diafragma; la segunda consiste en la contracción y relajación progresiva de todos los músculos del cuerpo, desde la parte superior del cuerpo hasta las regiones inferiores., divididos en 14 grupos. El proceso comienza con la respiración diafragmática y la sensibilización de todas las áreas de tensión en el cuerpo para finalizar con la evocación de la sensación de relajación en cada músculo. Los objetivos de la relajación son disminuir la hiperactivación simpática, el estrés psicológico, la ansiedad, e incrementar la percepción de autocontrol (Cea, 2010; Dornelas, 2008; Jensen \& Turk, 2014; Reynoso y Seligson, 2005). Además, la relajación favorece el involucramiento social, la asimilación de la información verbal durante la sesión y la percepción de bienestar (Domínguez, 2011; Porges, 2007; Porges, 2009).

- Psicoeducación: Tratamiento multimodal de autoayuda que utiliza información y estrategias cognitivo-conductuales para lograr cambios en el conocimiento y la conducta, con el objetivo de que el paciente consiga un autocontrol eficaz de su enfermedad (McGillion et al., 2008). El programa psicoeducativo se dividió en tres partes: 1) Insuficiencia cardiaca: definición, síntomas, diagnóstico y tratamiento; 2) Cambios en el estilo de vida y manejo de la enfermedad en casa; y 3 ) Planeación del futuro. 
- Técnica de solución de problemas: Consiste en ayudar al paciente a identificar y resolver problemas cotidianos que causan respuestas desadaptativas, encontrando soluciones alternativas a los problemas que le impiden llevar adecuadamente el tratamiento. Consta de cinco fases: 1) perfil del problema, 2) definición y formulación del problema, 3) generación de posibles soluciones o alternativas, 4) toma de decisiones, y 5) evaluación de los resultados (Reynoso y Seligson, 2005).

El objetivo fue promover conductas saludables y proporcionar herramientas que el paciente pudiera aplicar en su vida cotidiana para el manejo la IC. Se utilizó un registro diario de adherencia al tratamiento, una guía de ejercicios de Relajación Muscular Progresiva y la "Guía para pacientes con insuficiencia cardiaca", diseñada y avalada por un equipo interdisciplinario (cardiólogo, nutriólogo y psicólogo) especialistas en el tema especialmente y con amplio conocimiento de la población. La estructura general de cada sesión fue la siguiente:

Sesión 1. Presentación del(la) psicoterapeuta y de los participantes. Explicación general del programa de intervención. Explicación y práctica de la respiración diafragmática. Psicoeducación I: Insuficiencia cardiaca: principios básicos. Como tarea, los pacientes practicaban el ejercicio de relajación y el registro diario de adherencia al tratamiento de la IC.

Sesión 2. Práctica de la respiración diafragmática. Explicación y práctica de la relajación muscular progresiva. Psicoeducación II: Cambios en el estilo de vida y manejo de la IC en casa. Con base en el auto-registro de adherencia, se implementaba el entrenamiento en solución de problemas respecto a las principales dificultades cotidianas relacionados con el tratamiento. En casa los pacientes practicaban los ejercicios de relajación y realizaban la aplicación práctica de las alternativas de solución y el auto- registro.

Sesión 3. Práctica de relajación. Psicoeducación III: Planeando el futuro. Implementación y revisión de los resultados del entrenamiento en solución de problemas, identificación de nuevos problemas y búsqueda de soluciones. De tarea, los pacientes llevaban a cabo las técnicas aprendidas y el auto-registro.

Sesión 4. Práctica de relajación. Repaso y solución de dudas acerca de la intervención. Integración de la carpeta "Guía para pacientes con Insuficiencia cardiaca", con cada una de las secciones que se revisaron durante la psicoeducación. Cierre y despedida.

Para el caso del grupo control, los pacientes se encontraban en lista de espera para recibir el tratamiento psicológico una vez concluida la investigación. Finalmente, ambos grupos fueron evaluados con la prueba de caminata de seis minutos en una post-evaluación al término de la intervención y un seguimiento a tres meses.

\section{Resultados}

Los datos obtenidos se analizaron utilizando el programa SPSS para Windows, versión 19,0. Primero, se valoró el tipo de distribución de la muestra mediante la prueba Kolmogorov-Smirnov y gráficos Q-Q. A continuación, se realizaron análisis descriptivos de los datos sociodemográficos y la evaluación médica; además se calcularon las diferencias estadísticamente significativas entre las características de ambos grupos utilizando $t$ de student para muestras independientes para las variables numéricas y chi cuadrado $\left(\chi^{2}\right)$ para las variables categóricas.

Para calcular el efecto de la intervención se llevó a cabo un análisis de varianza de medidas repetidas para determinar cambios estadísticamente significativos entre ambos grupos en la distancia, además de prueba de Friedman para el nivel de esfuerzo y el nivel subjetivo de cansancio físico.

\section{Características sociodemográficas y clínicas}

No se detectaron diferencias estadísticamente significativas en las características sociodemográficas de ambos grupos; es decir, se trató de grupos estadísticamente equivalentes. En la Tabla 1 se pueden observar los datos correspondientes a cada grupo.

\section{Capacidad funcional}

Los cambios en la distancia recorrida durante la prueba de caminata de seis minutos muestran que el GC tuvo un incremento en la distancia en la post-evaluación, la cual disminuyó durante el seguimiento sin llegar a la registrada durante la pre-evaluación (Tabla 2). El GE tuvo un incremento paulatino en la distancia recorrida que llegó a ser estadísticamente significativo durante el seguimiento $(p=0,00)$.

En relación al NSCF reportado al finalizar la caminata, existieron diferencias estadísticamente significativas en la distribución del grupo experimental $(p=0,02)$. Por ejemplo, durante el seguimiento la mayoría de los pacientes reportó no tener cansancio o cansancio ligero (Tabla 2). Al respecto, el grupo control no reportó cambios estadísticamente significativos.

En cuanto al nivel de esfuerzo reportado por los pacientes al realizar la prueba de caminata, pese a que no hubo diferencias estadísticamente significativas, en el grupo experimental se incrementó el número de participantes que reportaron un esfuerzo muy muy ligero durante el seguimiento; al contrario del grupo control, donde se redujo esta proporción. Además en el grupo experimental ningún paciente volvió a reportar la categoría un poco pesado en las evaluaciones posteriores a la intervención (Tabla 4). 
Tabla 1. Características sociodemográficas y clínicas

\begin{tabular}{|c|c|c|c|c|}
\hline Variables & Control $(n=27)$ & Experimental $(\mathrm{n}=26)$ & Estadístico & p \\
\hline Edad & $\begin{array}{c}\tilde{x}_{=} \\
61.07 \pm 15.96 \\
(34-86)\end{array}$ & $\begin{array}{c}\tilde{x}_{=} 58.42 \pm 16.42 \\
(24-80)\end{array}$ & $t=0.596^{*}$ & 0.55 \\
\hline Género & & & $\chi^{2}=1.57$ & 0.21 \\
\hline Masculino & $15(55.6 \%)$ & $9(34.6 \%)$ & & \\
\hline Femenino & $12(44.4 \%)$ & $17(65.4 \%)$ & & \\
\hline Tiempo de diagnóstico & $\tilde{x}_{=3.35 \pm 0.98}$ & $\tilde{x}=3.01 \pm 1.28$ & $t=1.09^{*}$ & 0.28 \\
\hline Clase funcional & & & $\chi^{2}=0.00$ & 1.0 \\
\hline I & $18(66.7 \%)$ & $18(69.2 \%)$ & & \\
\hline ॥ & $9(33.3 \%)$ & $8(30.8 \%)$ & & \\
\hline
\end{tabular}

Tabla 2. Cambios en la distancia recorrida durante la prueba de caminata de seis minutos.

\begin{tabular}{|c|c|c|c|c|c|c|c|}
\hline & \multicolumn{3}{|c|}{ Grupo Control } & \multicolumn{3}{|c|}{ Grupo Experimental } & \multirow[b]{2}{*}{$\mathrm{F}$} \\
\hline & $\begin{array}{l}\text { Pre } \\
\tilde{\tilde{x}}_{(\mathrm{I}}^{\prime}=27 \\
{ }_{(\mathrm{DT})}\end{array}$ & $\begin{array}{l}\text { Post } \\
\ddot{\tilde{x}}_{(\mathrm{D}}^{\prime}=27 \\
{ }_{(\mathrm{DT})}\end{array}$ & $\begin{array}{l}\text { Seg } \\
\dot{\tilde{x}}_{(\mathrm{DT})}^{=21}\end{array}$ & $\begin{array}{l}\text { Pre } \\
\dot{\tilde{x}}_{(\mathrm{DT})}^{\prime}=26\end{array}$ & $\begin{array}{l}\text { Post } \\
\tilde{\tilde{x}}_{(\mathrm{DT})}^{\prime}=26\end{array}$ & $\begin{array}{l}\text { Seg } \\
\tilde{\tilde{x}}_{(\mathrm{d})}=25 \\
{ }_{(\mathrm{DT})}\end{array}$ & \\
\hline Distancia & $\begin{array}{l}297.5 \\
(107.1)\end{array}$ & $\begin{array}{l}317.7 \\
(111.6)\end{array}$ & $\begin{array}{l}305.1 \\
(74.3)\end{array}$ & $\begin{array}{l}253.9 \\
(80.4)\end{array}$ & $\begin{array}{l}255.91 \\
(77.8)\end{array}$ & $\begin{array}{l}310.7^{*} \\
(89.0)\end{array}$ & $\begin{array}{l}10.385 \\
{[2,40]}\end{array}$ \\
\hline
\end{tabular}

Abreviaturas. Pre: Pre-evaluación, Post: Post-evaluación, Seg: Seguimiento

Diferencias significativas: ${ }^{*} p=0.00$.

Tabla 3. Cambios del nivel subjetivo de cansancio físico.

\begin{tabular}{|c|c|c|c|c|c|c|c|c|}
\hline & \multicolumn{4}{|c|}{ Grupo Control } & \multicolumn{4}{|c|}{ Grupo Experimental } \\
\hline & $\begin{array}{c}\text { Pre } \\
\mathrm{N}=27\end{array}$ & $\begin{array}{l}\text { Post } \\
\mathrm{N}=27\end{array}$ & $\begin{array}{c}\text { Seg } \\
\mathrm{N}=21\end{array}$ & $\chi^{2}$ & $\begin{array}{c}\text { Pre } \\
\mathrm{N}=26\end{array}$ & $\begin{array}{c}\text { Post } \\
\mathrm{N}=26\end{array}$ & $\begin{array}{c}\text { Seg } \\
N=25\end{array}$ & $\chi^{2}$ \\
\hline Sin cansancio o fatiga & $6(22.2 \%)$ & $9(33.3 \%)$ & $4(19 \%)$ & 0.03 & $7(26.9 \%)$ & $10(38.5 \%)$ & $12(48.0 \%)$ & $7.5^{*}$ \\
\hline Ligeramente cansado & $13(48.1 \%)$ & $10(37.0 \%)$ & $11(52.4 \%)$ & & $11(42.3 \%)$ & $13(50.0 \%)$ & $11(44.0 \%)$ & \\
\hline Ni cansado, ni relajado & $2(7.4 \%)$ & $3(11.1 \%)$ & $1(4.8 \%)$ & & $2(7.7 \%)$ & $1(3.8 \%)$ & $1(4.0 \%)$ & \\
\hline $\begin{array}{l}\text { Moderadamente can- } \\
\text { sado }\end{array}$ & $5(18.5 \%)$ & $3(11.1 \%)$ & $4(19.0 \%)$ & & $6(23.1 \%)$ & $2(7.7 \%)$ & $1(4.0 \%)$ & \\
\hline Muy cansado y fatigado & $1(3.7 \%)$ & $2(7.4 \%)$ & $1(4.8 \%)$ & & & & & \\
\hline
\end{tabular}


Tabla 4. Cambios del nivel de esfuerzo.

\begin{tabular}{|c|c|c|c|c|c|c|c|c|}
\hline & \multicolumn{4}{|c|}{ Grupo Control } & \multicolumn{4}{|c|}{ Grupo Experimental } \\
\hline & $\begin{array}{l}\text { Pre } \\
\mathrm{N}=27\end{array}$ & $\begin{array}{l}\text { Post } \\
\mathrm{N}=27\end{array}$ & $\begin{array}{c}\text { Seg } \\
N=21\end{array}$ & $\chi^{2}$ & $\begin{array}{c}\text { Pre } \\
N=26\end{array}$ & $\begin{array}{l}\text { Post } \\
\mathrm{N}=26\end{array}$ & $\begin{array}{c}\text { Seg } \\
N=25\end{array}$ & $\chi^{2}$ \\
\hline Muy muy ligero & $\begin{array}{c}7 \\
(25.9 \%)\end{array}$ & $\begin{array}{c}6 \\
(22.2 \%)\end{array}$ & $\begin{array}{c}1 \\
(4.8 \%)\end{array}$ & 3.6 & $\begin{array}{c}3 \\
(11.5 \%)\end{array}$ & $\begin{array}{c}3 \\
(11.5 \%)\end{array}$ & $\begin{array}{c}8 \\
(32.0 \%)\end{array}$ & 3.9 \\
\hline Muy ligero & $\begin{array}{c}5 \\
(18.5 \%)\end{array}$ & $\begin{array}{c}5 \\
(18.5 \%)\end{array}$ & $\begin{array}{c}2 \\
(9.5 \%)\end{array}$ & & $\begin{array}{c}6 \\
(23.1 \%)\end{array}$ & $\begin{array}{c}3 \\
(11.5 \%)\end{array}$ & $\begin{array}{c}3 \\
(12.0 \%)\end{array}$ & \\
\hline Ligero & $\begin{array}{c}6 \\
(22.2 \%)\end{array}$ & $\begin{array}{c}4 \\
(14.8 \%)\end{array}$ & $\begin{array}{c}4 \\
(19.0 \%)\end{array}$ & & $\begin{array}{c}3 \\
(11.5 \%)\end{array}$ & $\begin{array}{c}5 \\
(19.2 \%)\end{array}$ & $\begin{array}{c}4 \\
(16.0 \%)\end{array}$ & \\
\hline Moderado & $\begin{array}{c}5 \\
(18.5 \%)\end{array}$ & $\begin{array}{c}3 \\
(11.1 \%)\end{array}$ & $\begin{array}{c}4 \\
(19.0 \%)\end{array}$ & & $\begin{array}{c}5 \\
(19.2 \%)\end{array}$ & $\begin{array}{c}5 \\
(19.2 \%)\end{array}$ & $\begin{array}{c}2 \\
(8.0 \%)\end{array}$ & \\
\hline Un poco pesado & $\begin{array}{c}1 \\
(3.7 \%)\end{array}$ & $\begin{array}{c}3 \\
(11.1 \%)\end{array}$ & $\begin{array}{c}2 \\
(9.5 \%)\end{array}$ & & $\begin{array}{c}2 \\
(7.7 \%)\end{array}$ & & & \\
\hline
\end{tabular}

Abreviaturas. Pre: Pre-evaluación, Post: Post-evaluación, Seg: Seguimiento. No se encontraron diferencias estadísticamente significativas.

Aun cuando el tratamiento multidisciplinario que sólo incluye los aspectos médico y nutricional coadyuvó para mantener la capacidad funcional de los pacientes del grupo control, la inclusión del tratamiento psicológico tuvo un efecto positivo en la capacidad funcional de los pacientes del grupo experimental: incrementó el tiempo invertido y la distancia recorrida, además de disminuir el nivel de esfuerzo.

\section{Discusión}

Los pacientes del grupo experimental incrementaron significativamente la distancia recorrida. De acuerdo con la literatura, este aumento se asocia con un mejor pronóstico, menor mortalidad, menor número de hospitalizaciones y mejor calidad de vida (Arslan et al., 2007; Forman et al., 2012; Guimarães et al., 2008; Jefferson et al., 2008; Rodríguez \& Rodríguez, 2005).

Más aún, al evaluar subjetivamente la experiencia de los pacientes al realizar la prueba de la caminata, se evidenció que los pacientes del grupo experimental disminuyeron sus niveles subjetivos de esfuerzo y cansancio en la post-evaluación y el seguimiento, este último de manera significativa. Lo anterior permite sugerir que, de acuerdo con la evidencia obtenida, los pacientes de este (i.e. los pacientes con IC sometidos al tratamiento) mejoraron su capacidad funcional.
Es preciso recordar que los pacientes fueron CF I y II, por lo que teóricamente no deberían tener limitaciones físicas al realizar esta prueba. Sin embargo, variables no consideradas en el tratamiento convencional de los pacientes, como las psicológicas, podrían estar influyendo en el desempeño de los pacientes en la prueba y explicar el cambio mostrado después de la intervención en el grupo experimental.

Un punto importante a destacar es el alto nivel de adherencia obtenido a lo largo del desarrollo del protocolo de investigación de parte de los pacientes que asistieron la intervención psicológica; sólo se perdió un paciente (3,8\%): por cirugía de trasplante renal, motivo por el cual tuvo que permanecer en aislamiento y no fue posible realizar la evaluación correspondiente. En México, la falta de adherencia a los tratamientos psicológicos integrados en sesiones numerosas es un problema grave; este estudio mostró, además, que una intervención breve favoreció la adherencia de los pacientes a la terapia psicológica.

Durante la impartición de la intervención psicológica se observó la creación de relaciones de apoyo entre los participantes, por lo que una posible explicación para el nivel de adherencia registrado es el apoyo social percibido al formar parte del grupo de intervención, que pudo reforzar la adherencia al tratamiento y la asistencia a las citas de evaluación, así como el incremento del efecto 
de la intervención durante el seguimiento. No obstante, dado que esta variable no fue evaluada, se recomienda su inclusión en futuros protocolos de investigación, además de variables fisiológicas que respalden el cambio a nivel clínico.

Con base en la evidencia encontrada es posible concluir que la intervención psicológica propuesta se adecua a las necesidades específicas de la población a la cual se encuentra dirigida. Adicionalmente, brinda elementos para considerar nuevas líneas de investigación que indaguen y promulguen la importancia de incorporar este tipo de intervenciones en los protocolos de atención de pacientes con este síndrome, de modo puedan emplearse como tratamientos complementarios y reforzadores de los tratamientos farmacológicos y de las Guías de Práctica Clínica para el Diagnóstico y Tratamiento tradicionales.

Se recomiendan estudios futuros que incluyan tamaños de muestra mayores, seguimientos a largo plazo, e inclusión de otras variables psicosociales y fisiológicas que puedan mediar el proceso salud-enfermedad en los pacientes con IC. Asímismo se resalta la importancia de la integración de equipos de evaluación, tratamiento e investigación multidisciplinarios que repercutan en el mejoramiento de la salud cardiovascular y la calidad de vida de los pacientes.

Agradecimiento al Consejo Nacional de Ciencia y Tecnología (CONACyT) [Beca doctoral 262555] y Proyecto CONACYT-FOSISS: 262266 "Efecto del tratamiento no farmacológico sobre el estado clínico y pronóstico del paciente con Insuficiencia Cardíaca y Respiratoria", sin conflicto de intereses.

\section{Referencias}

Achury, S.D. (2007). Autocuidado y adherencia en pacientes con falla pacientes con falla cardiaca. Chía, 7(2), 139160.

Alarcón, R., y Ramírez V.E. (2006b). Medicina psicosomática en enfermedad cardiovascular II. Consideraciones sobre el tratamiento. Revista Colombiana de Psiquiatría, 35 (3), 362-379.

Alfaro, M., Asenjo, R., Castro, P., Jalil, J., LLancaqueo, M., Sepúlveda, L. \& Vukasovic, J. (2007). Guías para el diagnóstico y tratamiento de la Insuficiencia Cardiaca. Revista Chilena de Cardiología, 26(4), 473-504.

Arrivillaga, Q.M., Varela, A.M., Cáceres, R.D., Correa S.D. y Holguín, P.L. (2007). Eficacia de un programa cognitivo conductual para la disminución de los niveles de presión arterial. Pensamiento Psicológico, 3(9), 33-49.

Arslan, S., Erol, M. K., Gundogdu, F., Sevimli, S., Aksakal, E., Senocak, H., \& Alp, N. (2007). Prognostic value of 6-minute walk test in stable outpatients with heart failure. Texas Heart Institute Journal. 34(2), 166-169.

Asensio E., Orea, A., Castillo L., Narváez R., Dorantes, J. y
Oseguera, J. (2005). Utilidad de la estimulación eléctrica en la insuficiencia cardiaca. Medicina Interna de México, 21(1), 52-59.

Cea, U.J. (2010). El arte de la relajación: bases funcionales de la relajación mediante respiración manejada a voluntad. Advances in Relational Mental Health, 9(1), 15793516.

Cerezo, R.S., Hernández, P.M., y Rodríguez, O.G. (2009). Efectos de una intervención de manejo el estrés en mujeres con hipertensión arterial sistémica. Acta Colombiana de Psicología, 12(1), 85-93.

Del Pino, A., Gaos, M., Dorta R. y García M. (2004). Eficacia de un programa cognitivo-conductual para modificar conductas prono-coronarias. Psicothema, 16(4), 654660.

Domínguez, T.B. (2011) El sistema nervioso autónomo y nuestra capacidad para relacionarnos. Enseñanza e Investigación en Psicología, 12(2), 5-51.

Dornelas, E.A. (2008). Psychotherapy with cardiac patients. Behavioral cardiology in practice. Washington DC: American Psychological Association.

Forman, D. E., Fleg, J. L., Kitzman, D. W., Brawner, C. A., Swank, A. M., McKelvie, R. S., \& Bittner, V. (2012). 6-Min Walk Test Provides Prognostic Utility Comparable To Cardiopulmonary Exercise Testing in Ambulatory Outpatients With Systolic Heart Failure. Journal of the American College of Cardiology, 60(25), 2653-61. doi:10.1016/j. jacc.2012.08.1010

Guimarães, G. V., Carvalho, V. O., \& Bocchi, E. A. (2008). Reproducibility of the self-controlled six-minute walking test in heart failure patients. Clinics (Sao Paulo, Brazil), 63(2), 201-206. doi:10.1590/S1807-59322008000200008

Hunt, S. A., Abraham, W. T., Chin, M. H., Feldman, A. M., Francis, G. S., Ganiats, T. G., \& Yancy, C. W. (2009). 2009 Focused update incorporated into the ACC/AHA 2005 Guidelines for the Diagnosis and Management of Heart Failure in Adults A Report of the American College of Cardiology Foundation/American Heart Association Task Force on Practice Guidelines Developed . Journal of the American College of Cardiology, 53(15), e1-e90. doi:10.1016/j.jacc.2008.11.013

Jefferson, J. A., Santos, P.T., Paulo R. S. \& Brofman M.D. (2008). Six-minute walk test and quality-of-life in heart failure. A correlative study with a Brazilian sample. Insuficiencia Cardiaca, 3(2), 76-79.

Jensen, M.P. \& Turk, D.C. (2014). Contributions of psychology to the understanding and treatment of people with chronic pain. American Psychologist, 69(2), 105-118. doi: 10.1037/a0035641.

Jiménez, S.C., Luna, S.M. y Huertas, N.E. (2009). Diagnóstico y tratamiento de la insuficiencia cardiaca aguda. México: Secretaría de Salud.

Kulur, A. B., Haleagrahara, N., Adhikary, P., \& Jeganathan, P. S. (2009). Effect of diaphragmatic breathing on heart rate variability in ischemic heart disease with diabetes. 
Arquivos Brasileiros de Cardiologia, 92(6), 423-463. doi:10.1590/S0066-782X2009000600008

McGillion, M. H., Watt-Watson, J., Stevens, B., LeFort, S. M., Coyte, P., \& Graham, A. (2008). Randomized ControIled Trial of a Psychoeducation Program for the Self-Management of Chronic Cardiac Pain. Journal of Pain and Symptom Management, 36(2), 126-140. doi:10.1016/j. jpainsymman.2007.09.015

McMurray, J. J. V., Adamopoulos, S., Anker, S. D., Auricchio, A., Böhm, M., Dickstein, K., \& Zeiher, A. (2012). ESC Guidelines for the diagnosis and treatment of acute and chronic heart failure 2012: The Task Force for the Diagnosis and Treatment of Acute and Chronic Heart Failure 2012 of the European Society of Cardiology. Developed in collaboration with the Heart. European Heart Journal, 33, 1787-847. doi:10.1093/eurheartj/ehs104

Orea, T.A., y Jiménez, D.V. (2005). Epidemiología de la insuficiencia cardiaca. Magnitud del problema. In A. Orea-Tejeda, L. Castillo-Martínez, \& C. Rodríguez-Gilabert (Eds.), Factores de Riesgo Cardiovascular e Insuficiencia Cardiaca (1st ed., pp. 235-246). México: McGraw-Hill Interamericana.

Porges, S.W. (2007). The Polyvagal Perspective. Biological Psychology, 74(2), 116-143. doi: 10.1016/j.biopsycho.2006.06.009
Porges, S.W. (2009) The polyvagal theory: New insights into adaptive reactions of the autonomic nervous system. Cleveland Clinic Journal of Medicine, 76(2), 86-90. doi:10.3949/ccjm.76.s2.17.

Reyes, C.L. (2011). Intervención cognitivo-conductual para la disminución del estrés en pacientes con cardiopatía isquémica. Summa Psicológica, 8(1), 21-28.

Reynoso, E.L. y Seligson N.I. (2005). Psicología clínica de la salud. Un enfoque conductual. México: El Manual Moderno.

Rodríguez, G.C., y Rodríguez, D.G. (2005). Valoración de la función del sistema cardiovascular como predictor de riesgo en pacientes con insuficiencia cardiaca. In A. Orea Tejeda, L. Castillo Martínez, \& C. Rodríguez Gilbert (Eds.), Factores de riesgo cardiovasculares e Insuficiencia Cardiaca (pp. 155-164). México: McGraw-Hill Interamericana.

Roger, V. L., Go, A. S., Lloyd-Jones, D. M., Benjamin, E. J., Berry, J. D., Borden, W. B., \& Turner, M. B. (2012). Heart disease and stroke statistics--2012 update: a report from the American Heart Association. Circulation, 125(1), e2e220. doi:10.1161/CIR.0b013e31823ac046.

Rubin, S. A. (2012). Exercise tests in heart failure patients: is simple better? Journal of the American College of Cardiology, 60(25), 2662-3. doi:10.1016/j.jacc.2012.08.1012 